Supporting Information

\title{
Multi-ion Modulated Single-Step Synthesis of a Nanocarbon Embedded with a Defect-Rich Nanoparticle Catalyst for a High Loading Sulfur Cathode
}

Jian Wang ${ }^{1,2}$, Lujie Jia ${ }^{3}$, Haitao $\mathrm{Liu}^{4}$, Chong Wang ${ }^{3}$, Jun Zhong ${ }^{5}$, Qingbo Xiao², Jin Yang ${ }^{2}$, Shaorong Duan ${ }^{3}$, Kun Feng ${ }^{5}$, Na Liu², Wenhui Duan ${ }^{3}$, Hongzhen Lin ${ }^{1,2}$ \& Yuegang Zhang $*, 2,3$

${ }^{1}$ School of Nano-Technology and Nano Bionics, University of Science and Technology of China, Hefei, Anhui, 230026, China

${ }^{2} i$-Lab, Suzhou Institute of Nano-Tech and Nano-Bionics, Chinese Academy of Sciences, Suzhou, Jiangsu, 215123, China

${ }^{3}$ State Key Laboratory of Low Dimensional Quantum Physics and Department of Physics, Tsinghua University, Beijing, 100084, China

${ }^{4}$ Institute of Applied Physics and Computational Mathematics, Beijing, 100088, China

${ }^{5}$ Institute of Functional Nano and Soft Materials Laboratory (FUNSOM), Soochow University, Suzhou, Jiangsu, 215123, China

Corresponding author: yuegang.zhang@tsinghua.edu.cn 

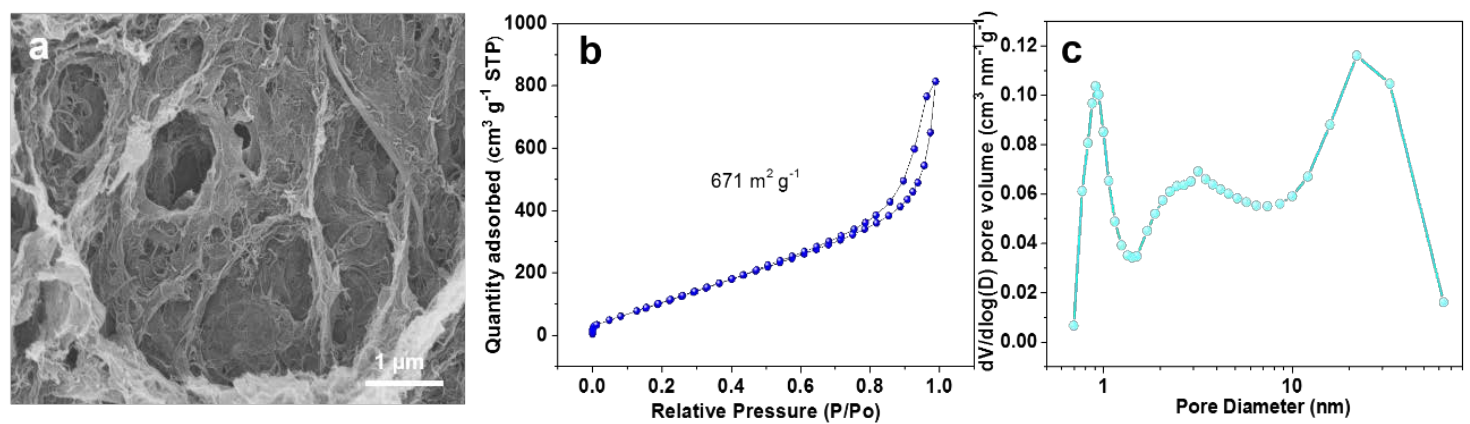

Figure S1. (a) A typical SEM image of the 3DGC matrix. (b) Nitrogen adsorption/desorption isotherm and (c) the pore distribution curve of the porous 3DGC.
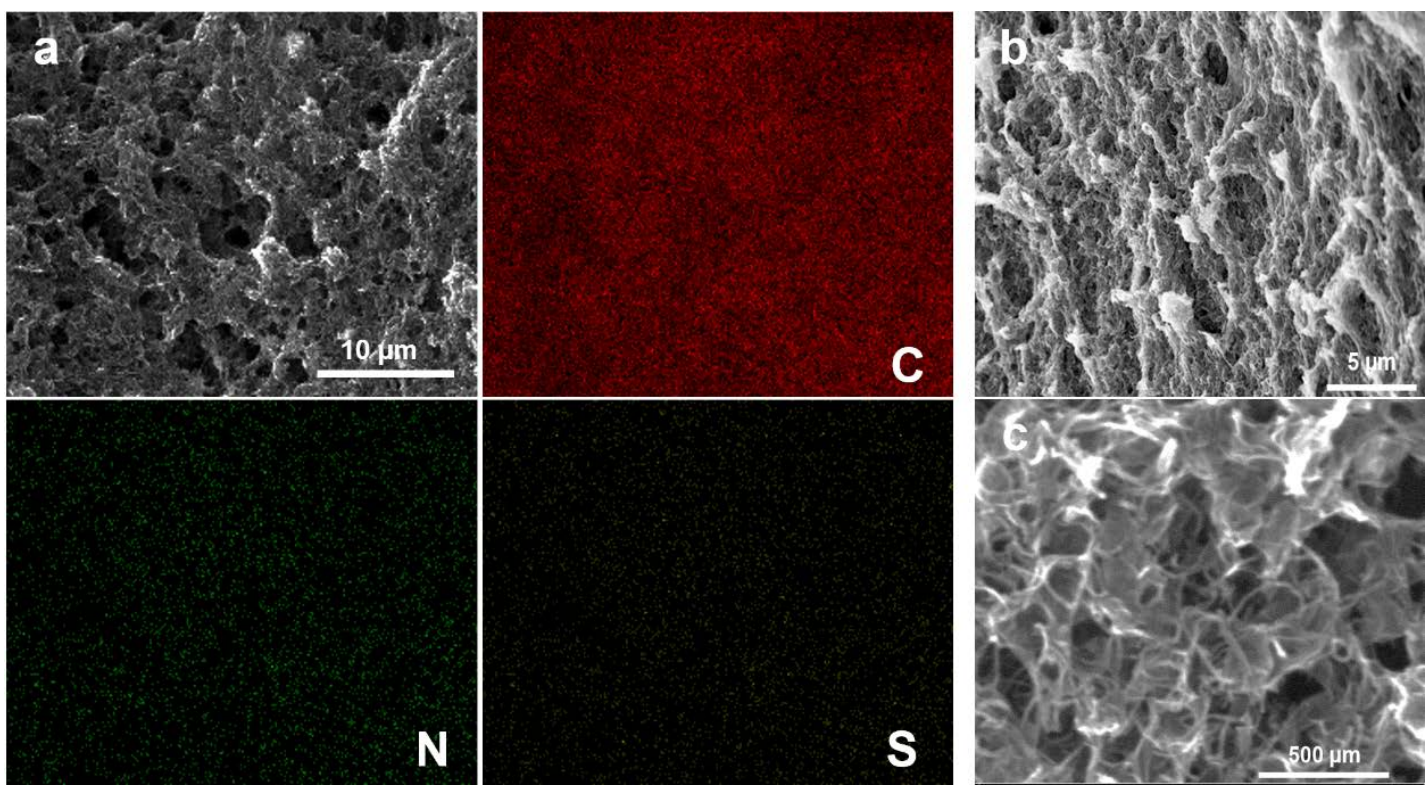

Figure S2. (a) EDS mapping of different elements (C, N, and S) in the 3DGC matrix. The (b) low-resolution and (c) high-resolution SEM images of ODFO@3DGC nanocomposites. 

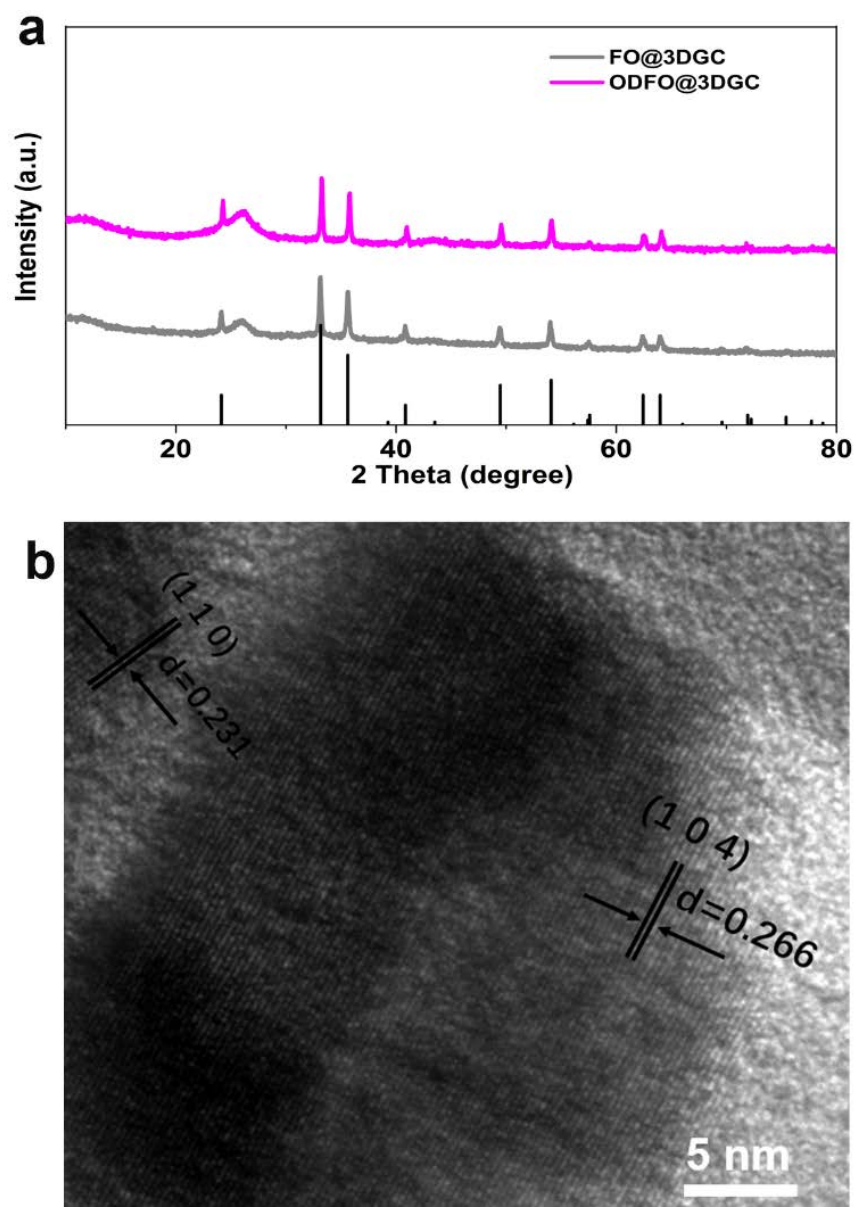

Figure S3. (a) XRD patterns of the ODFO@3DGC and FO@3DGC nanocomposites. (b) The high-resolution TEM image of ODFO@3DGC nanocomposite. 

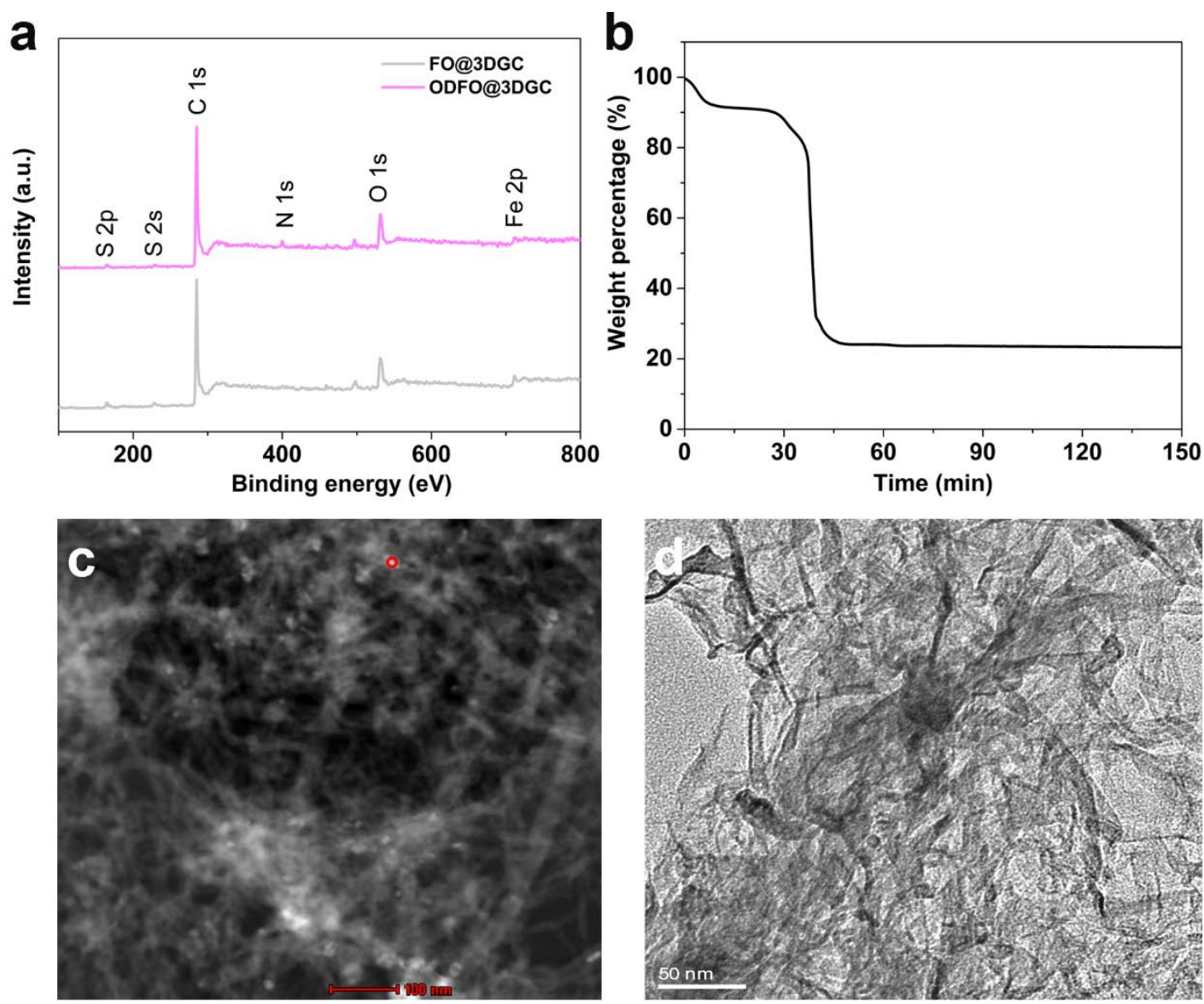

Figure S4. (a) The wide XPS surveys of the FO@3DGC and ODFO@3DGC composites.

(b) TG curve of the ODFO@3DGC composite with a heating rate of $10^{\circ} \mathrm{C} \min ^{-1}$ to $800{ }^{\circ} \mathrm{C}$ and kept at $800{ }^{\circ} \mathrm{C}$ for about 70 min under the atmosphere of dry air. The TEM images of (c) FO@3DGC and (d) 3DGC nanocomposites. 

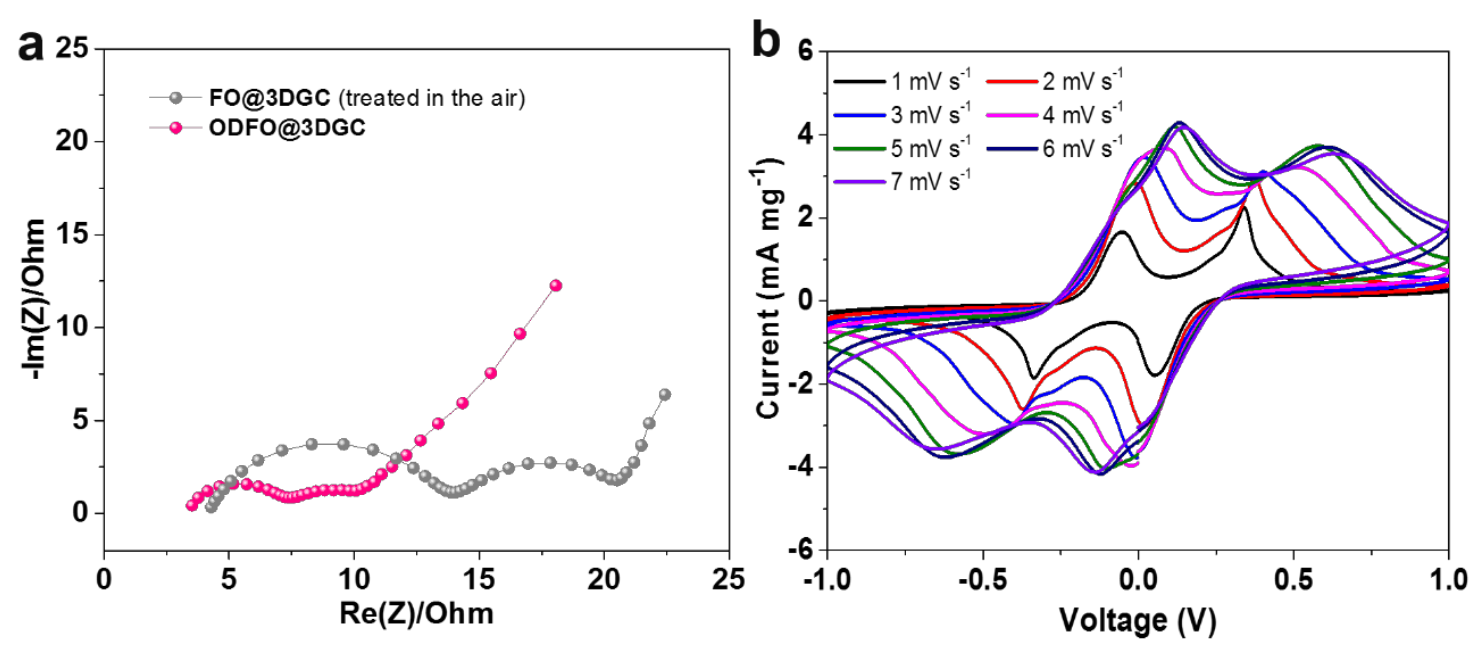

Figure S5. (a) EIS comparison between the ODFO@3DGC and FO@3DGC symmetric cells. (b) CV profiles of the FO@3DGC symmetric cell in the potential range from -1 to 1 $\mathrm{V}$ at various scan rates.
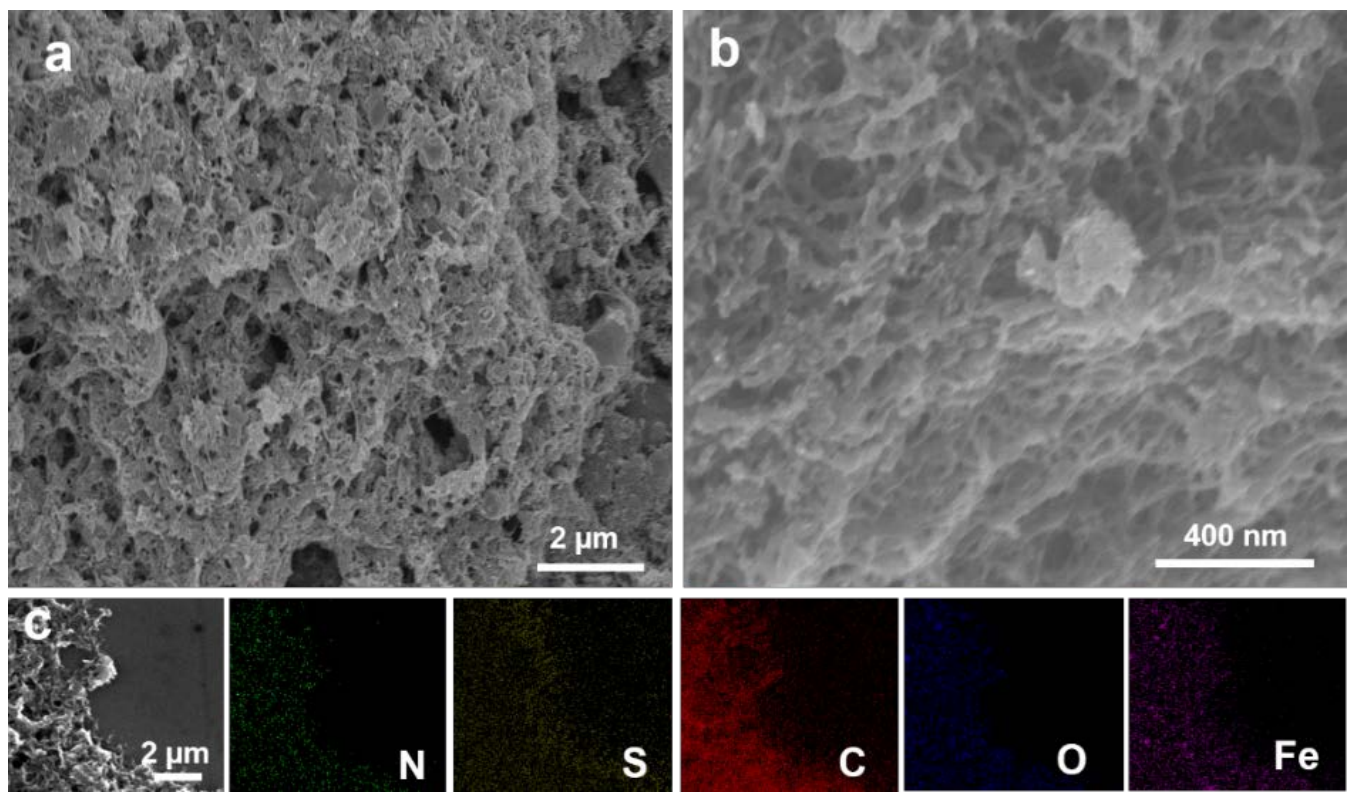

Figure S6. The (a) low-resolution and (b) high-resolution SEM images and (c) elemental mapping images of the ODFO@3DGC@S composite.

After the sulfur infusion process, the ODFO@3DGC@S composite still maintains the 3D porous morphology and the sulfur is uniformly distributed without showing any observable sulfur islands (Figure S6). 

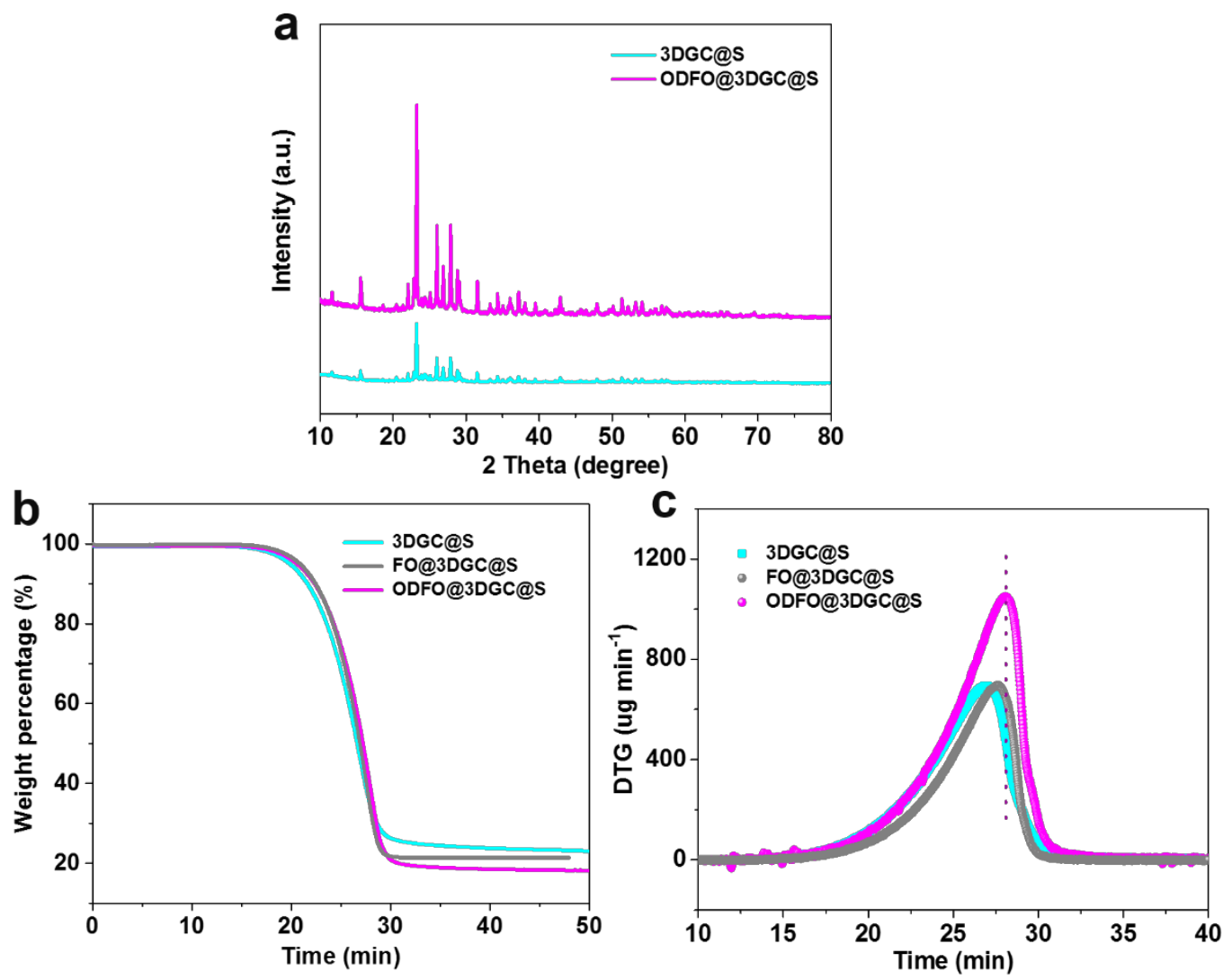

Figure S7. XRD patterns of (a) the different sulfur cathode nanocomposites. (b) TG curves and (c) DTG curves of the as-fabricated three cathode nanocomposites with a heating rate of $10{ }^{\circ} \mathrm{C} \min ^{-1}$ to $500{ }^{\circ} \mathrm{C}$ under the atmosphere of nitrogen gas.

The high specific surface area of the porous matrix ensures a high loading of sulfur (Figure S7a). Based on TG analysis, the sulfur content is determined to be $80 \%$, $78 \%$ and $76 \%$ below500 ㄷ for ODFO@3DGC@S, FO@3DGC@S and 3DGC@S composites, respectively (Figure S7b). Moreover, the ODFO@3DGC@S system requires higher temperature than the other two (Figure S7c), implying that the ODFO@3DGC matrix has stronger ability to accommodate and immobilize sulfur. 

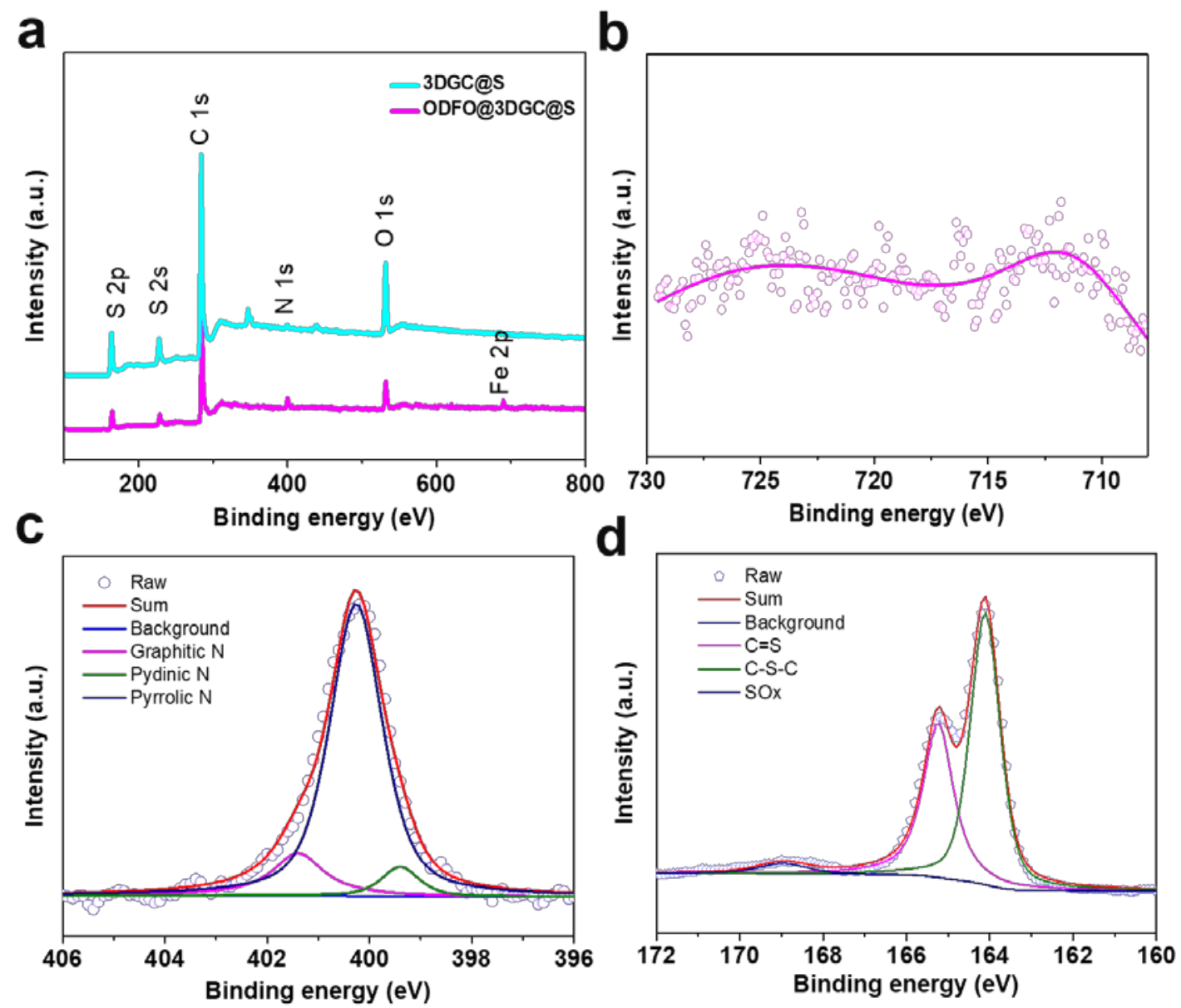

Figure S8. (a) XPS full scan spectra of ODFO@3DGC@S and 3DGC@S. The highresolution XPS of (b) $\mathrm{Fe}_{2 p}$, (c) $\mathrm{N}_{1 \mathrm{~s}}$ and (d) $\mathrm{S}_{2 \mathrm{p}}$ spectra in the ODFO@3DGC@S composites.

The XPS signals of Fe element become extremely weak (Figure S8a-8b), indicating that the ODFO nanoclusters are mostly buried inside by the sulfur. The nitrogen and sulfur functional groups are also detected in Figure S8c-8d, which are beneficial for the adsorption of polysulfides. 


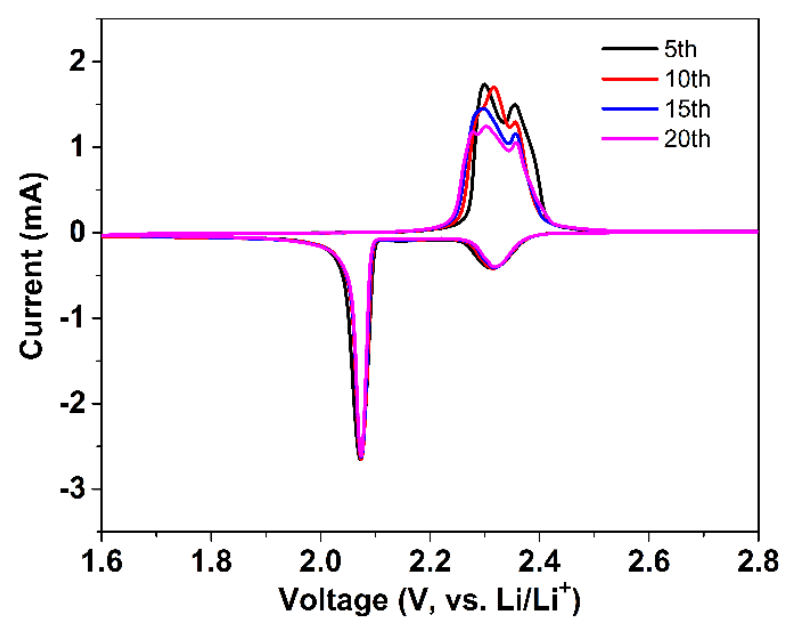

Figure S9. CV profiles of ODFO@3DGC@S at a scan rate of $0.05 \mathrm{mV} \mathrm{s}^{-1}$.
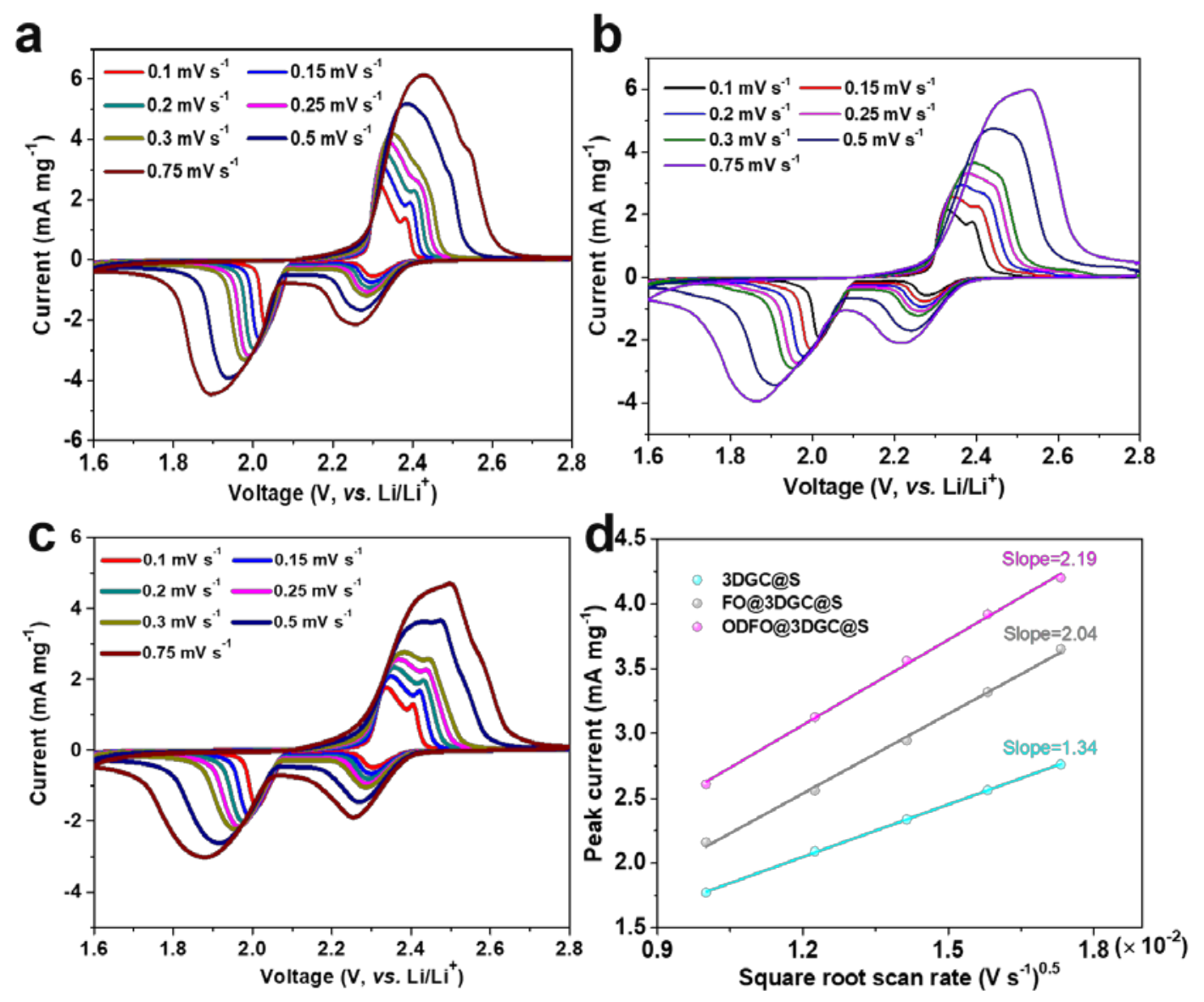

Figure S10. The CV curves of (a) ODFO@3DGC@S, (b) FO@3DGC@S, and (c) 3DGC@S at different scan rates between 1.6 and 2.8 V. (d) Plot of the peak current for the first anodic process $\left(\mathrm{Li}_{2} \mathrm{~S} \leftrightarrow \mathrm{Li}_{2} \mathrm{~S}_{\mathrm{x}}\right)$ as a function of the square root of the scan rates. 

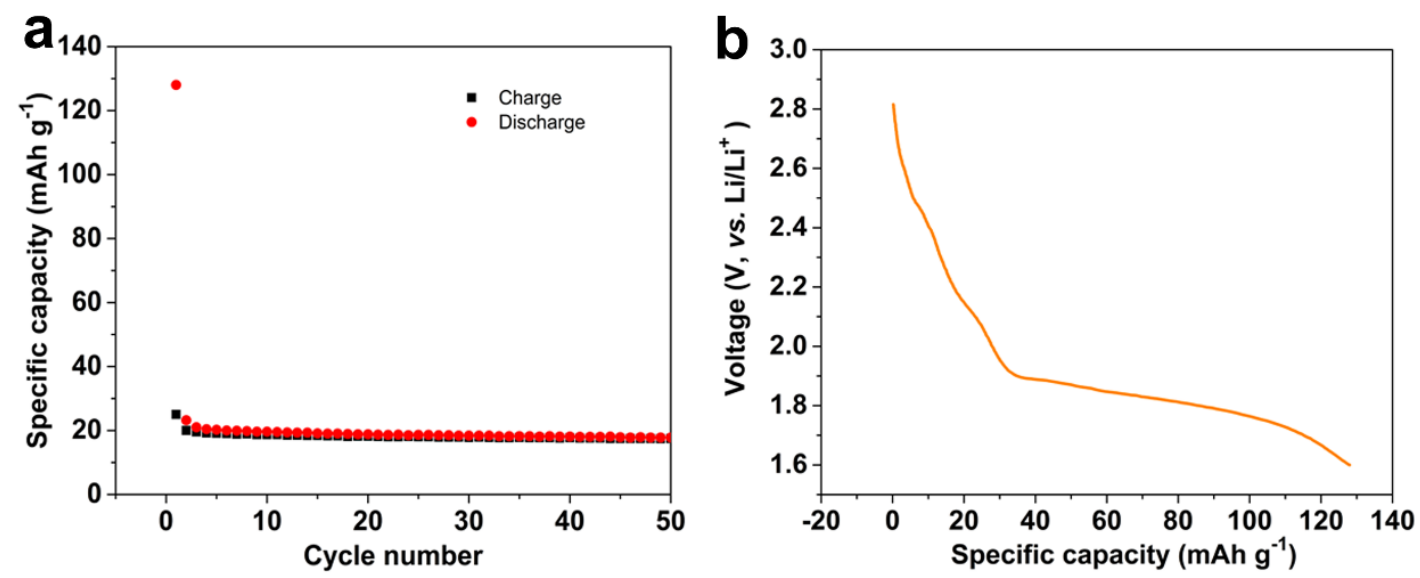

Figure S11. (a) Cycling performance of the ODFO@3DGC cathode at 0.5C. (b) The initial discharge profile of ODFO@3DGC cathode.

To evaluate the capacity contribution from the matrix, the ODFO@3DGC cathode's electrochemical performance without sulfur has been tested with the same method as a blank controlled experiment. As displayed in Figure S11, the initial discharge capacity of pristine ODFO@3DGC electrode is only about $100 \mathrm{~mA} \mathrm{~h} \mathrm{~g}^{-1}$ at $0.05 \mathrm{C}$ and only affords 32 $\mathrm{mA} \mathrm{h} \mathrm{g}{ }^{-1}$ at $0.5 \mathrm{C}$. This fact indicates that the iron oxide loaded carbon materials, and the partial decomposition of the additive $\left(\mathrm{LiNO}_{3}\right)$, if any, afford very little lithiation capacity. 

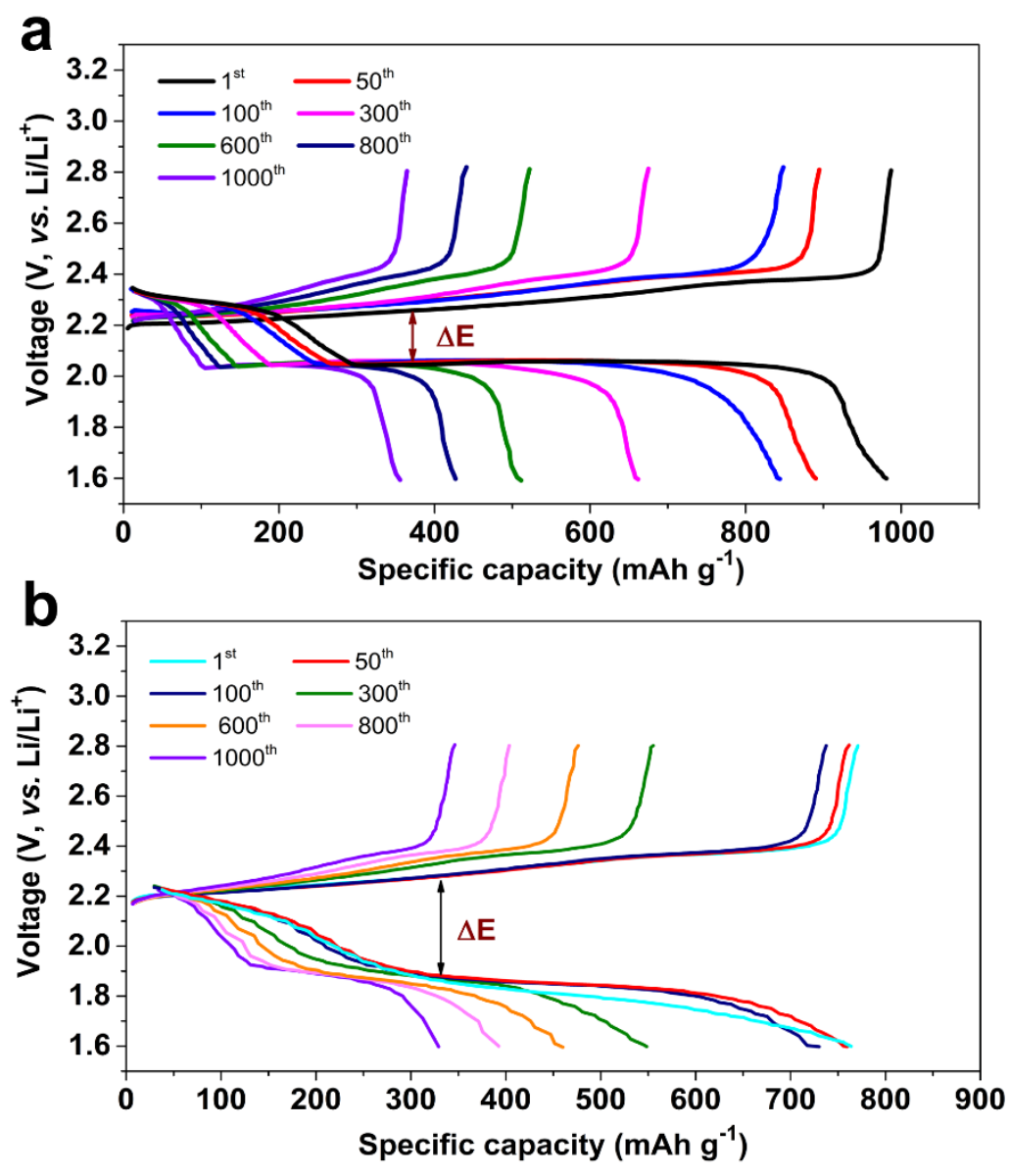

Figure S12. The voltage profiles of different cycles (a) discharged at $2 \mathrm{C}$ and (b) $10 \mathrm{C}$ and charged at 2 C, respectively, suggest nearly unchanged polarization in the ODFO@3DGC@S cathode for 1000 cycles. 


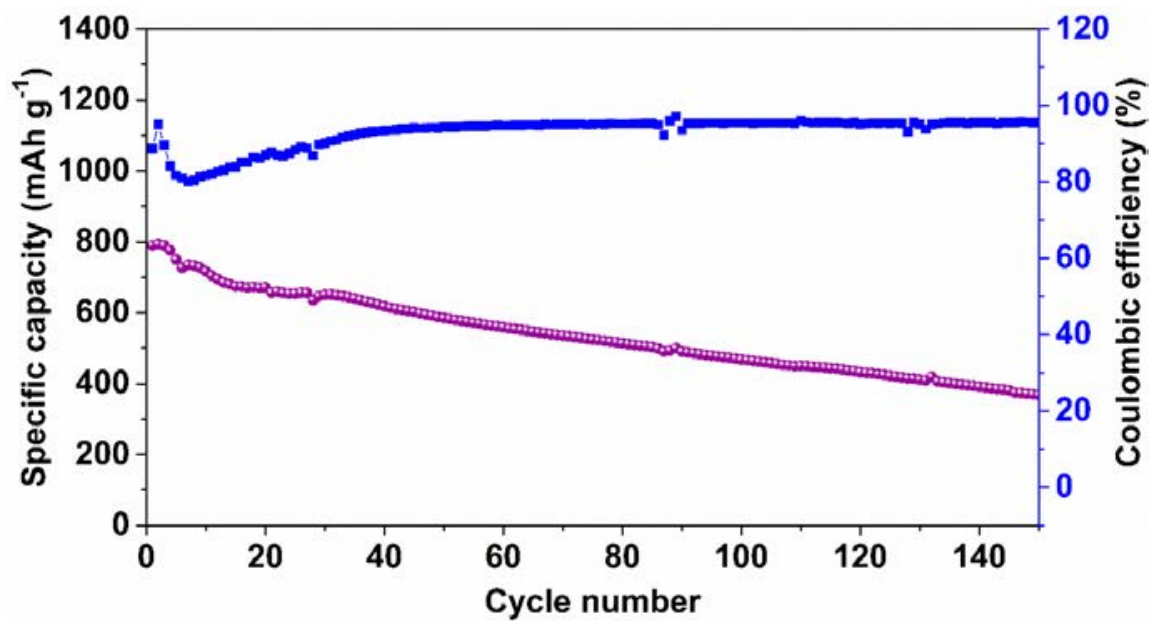

Figure S13. The cycling performance of ODFO@3DGC@S cathode with high loading of $11.02 \mathrm{mg} \mathrm{cm}^{-2}$ at $0.5 \mathrm{C}$.

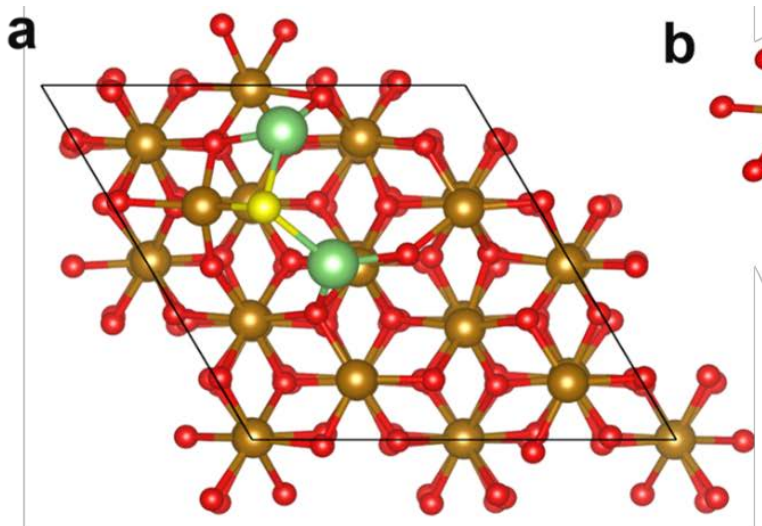

C

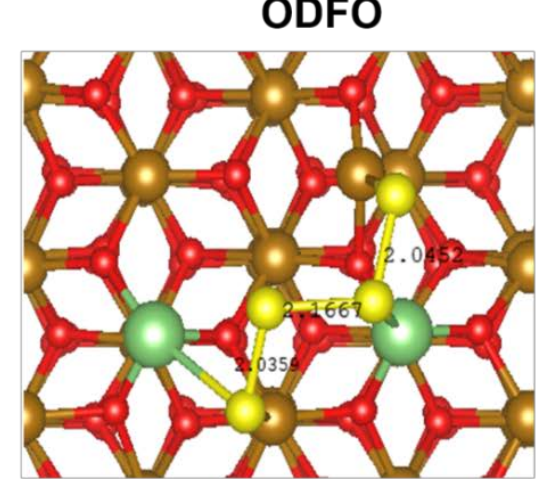

d

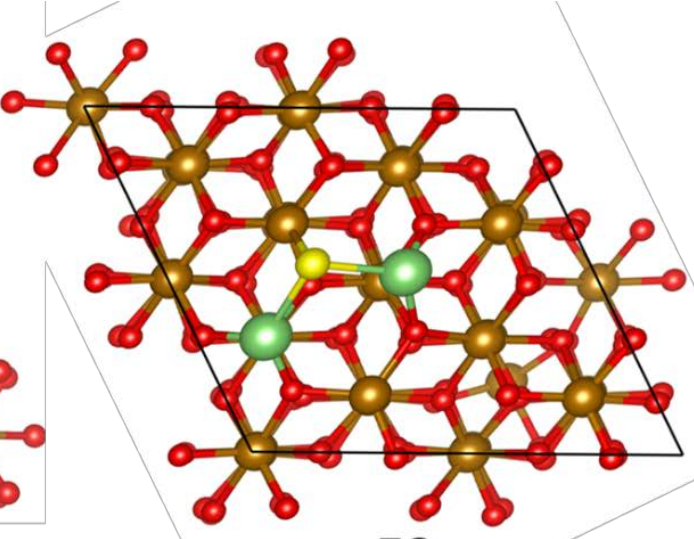

FO

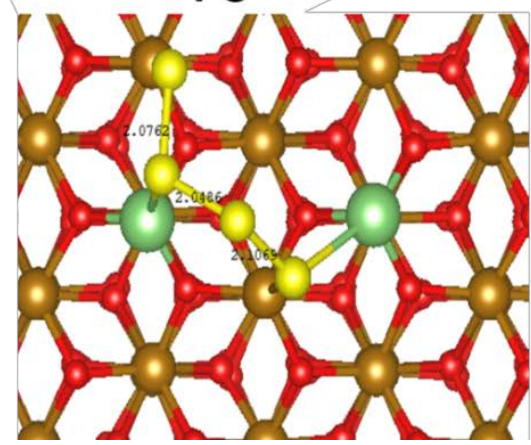

Figure 14. Theoretical simulation between $\mathrm{Li}_{2} \mathrm{~S}$ and (a) ODFO and (b) FO catalysts surface in top view side. The S-S bond length variation in $\mathrm{Li}_{2} \mathrm{~S}_{4}$ on the surface of (c) ODFO and (d) FO, respectively. 
Table S1. Electrocatalytic parameters for these three sulfur electrodes are derived from cyclic voltammograms and potentiostatic polarization

\begin{tabular}{|c|c|c|c|c|}
\hline Electrodes & Ep, $\mathbf{r}_{1} \& E p, \mathbf{r}_{2}(\mathbf{V})$ & Ep, $\mathbf{o}_{1} \& E p, \mathbf{o}_{2}(\mathbf{V})$ & $\begin{array}{c}\text { Peak separation } \\
(\mathbf{m V}) \\
\text { Ep, } \mathbf{o}_{2} \sim \text { Ep, } \mathbf{r}_{\mathbf{1}}\end{array}$ & $\begin{array}{c}\text { Exchange current } \\
\text { density }\left(\mathbf{m A ~ c m}^{-2}\right)\end{array}$ \\
\hline ODFO@3DGC@S & $2.058 \& 2.300$ & $2.318 \& 2.358$ & 300 & 1.09 \\
\hline FO@3DGC@S & $2.047 \& 2.297$ & $2.335 \& 2.380$ & 333 & 0.293 \\
\hline 3DGC@S & $2.012 \& 2284$ & $2.348 \& 2.396$ & 384 & 0.121 \\
\hline
\end{tabular}

Table S2. Comparison of the overall electrochemical performance of different catalysts in lithium-sulfur batteries.

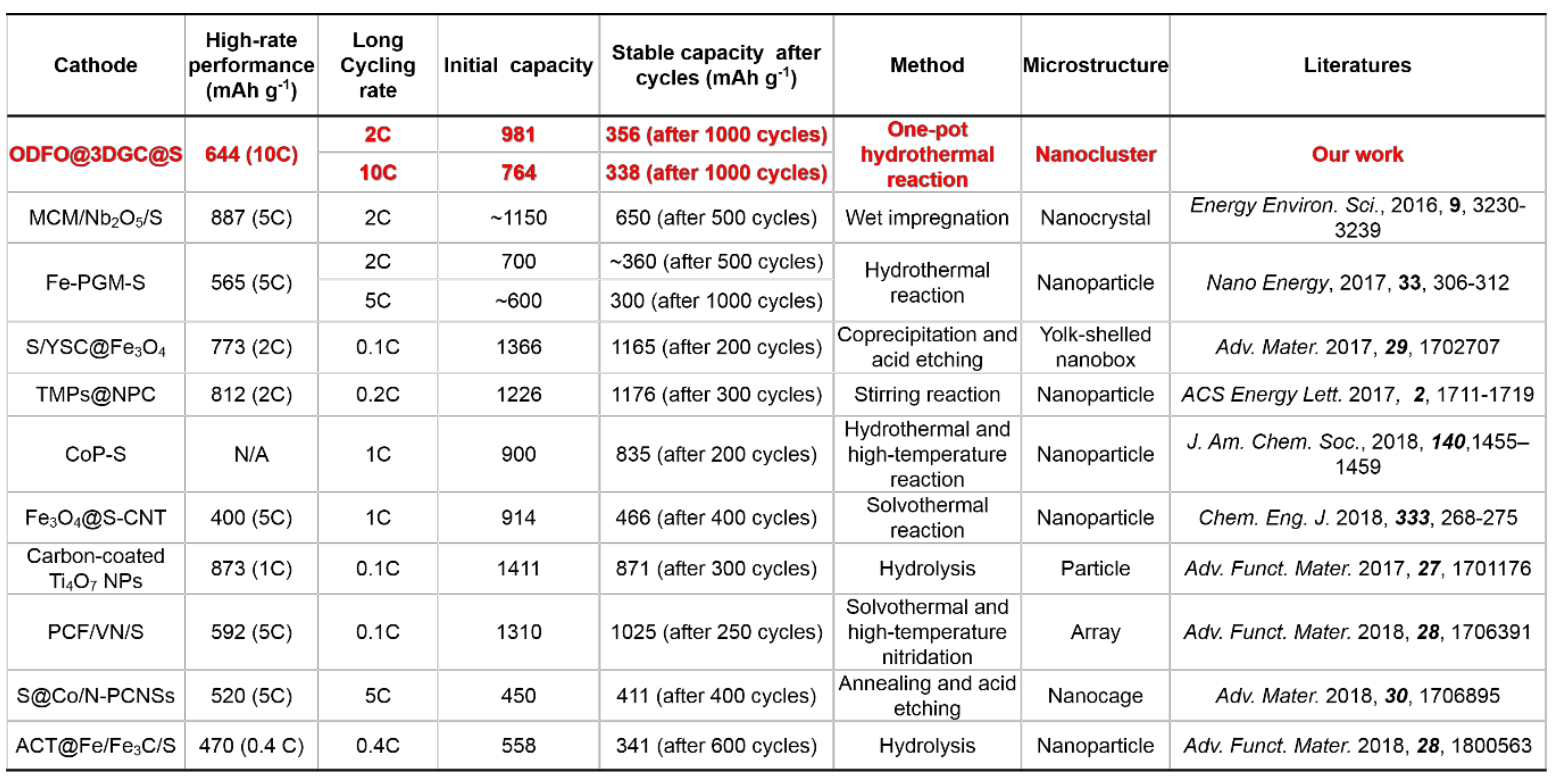


Table S3. Comparison of the areal capacity performance of different loading in lithiumsulfur batteries. (Note: These values are collected after 100 cycles at various current rate.)

\begin{tabular}{|c|c|c|c|}
\hline $\begin{array}{c}\text { Areal loading } \\
\left(\mathbf{m g ~ c m}^{-2}\right)\end{array}$ & Current rate & $\begin{array}{c}\text { Specific capacity } \\
\left(\mathbf{m A h} \mathbf{~ g}^{-1}\right)\end{array}$ & \multicolumn{2}{c|}{ Oiterature } \\
\hline 11.02 & $0.5 \mathrm{C}$ & 790 (initial) & Our work \\
\hline 11.02 & $0.5 \mathrm{C}$ & 468 & Nano Lett. 2017, 17,7086 \\
\hline 4.2 & $\sim 0.1 \mathrm{C}$ & $\sim 650$ & Adv. Energy Mater. 2017,7,1601943 \\
\hline 4.6 & $0.5 \mathrm{C}$ & 800 & Adv. Mater. 2017,29,1603040 \\
\hline 5.5 & $0.2 \mathrm{C}$ & 600 & Nano Energy 2018,51,73 \\
\hline 7.1 & $0.2 \mathrm{C}$ & 600 & Adv. Energy Mater. 2017,7,1602347 \\
\hline 8.1 & $0.5 \mathrm{C}$ & 650 & \\
\hline
\end{tabular}

